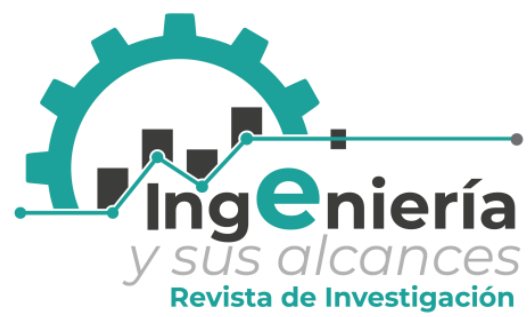

\title{
Sistema administrativo para optimizar la gestión académica de la red universitaria Domingo Savio
}

\author{
Administrative system to optimize the academic management of the Domingo \\ Savio university network
}

\author{
Sistema administrativo para otimizar a gestão acadêmica da rede \\ universitária Domingo Savio
}

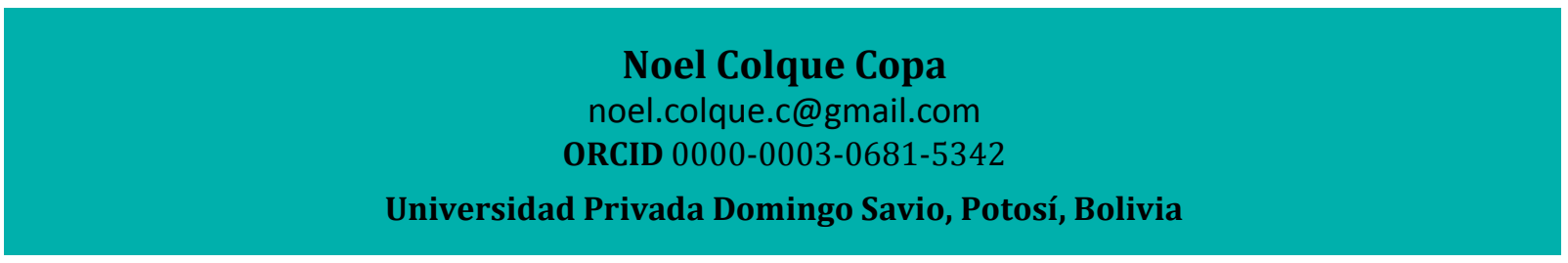

Artículo recibido mayo 2020 | Arbitrado en julio 2020 | Publicado en septiembre 2020

\section{RESUMEN}

El objetivo de la investigación es el desarrollo de un sistema administrativo fundamentado en la tecnología XP que permita optimizar la gestión académica en la red de la Universidad Privada Domingo Savio (UPDS). Se parte de un diagnóstico de necesidades realizado recogiendo información de los directivos y el personal docente. El objeto de estudio es la gestión académica, por su naturaleza se clasifica como una investigación proyectiva tecnológica, el diseño es de campo. Las técnicas de recolección de datos son la observación, la encuesta y la entrevista y los instrumentos la guía de observación, el cuestionario y el guion de entrevista. Como resultado se presenta el sistema administrativo para la gestión académica que se construyó aplicando la Metodología Ágil XP, que permite dar respuesta a las necesidades de la organización universitaria.

Palabras clave: Sistema administrativo; gestión académica; Universidad Privada Domingo Savio; Metodología Ágil XP
ABSTRACT

RESUMO
The objective of the research is the development of an administrative system based on XP technology that allows optimizing academic management in the network of the Domingo Savio Private University (UPDS). It starts from a needs assessment carried out by collecting information from managers and teaching staff. The object of study is academic management, by its nature it is classified as a technological projective research, the design is field. The data collection techniques are the observation, the survey and the interview and the instruments the observation guide, the questionnaire and the interview script. As a result, the administrative system for academic management that was built applying the Agile XP Methodology is presented, which allows responding to the needs of the university organization.

Key words: Administrative system; academic management; Domingo Savio Private University; Agile XP Methodology
0 objetivo da pesquisa é o desenvolvimento de um sistema administrativo baseado na tecnologia XP que permita otimizar a gestão acadêmica na rede da Universidade Privada Domingo Savio (UPDS). Partese de uma avaliação das necessidades realizada por meio da coleta de informações junto aos gestores e docentes. 0 objeto de estudo é a gestão acadêmica, por sua natureza é classificada como uma pesquisa projetiva tecnológica, o design é de campo. As técnicas de recolha de dados são a observação, o inquérito e a entrevista e os instrumentos o guia de observação, o questionário e o guião de entrevista. Como resultado, é apresentado o sistema administrativo de gestão acadêmica que foi construído aplicando a Metodologia Agile XP, que permite responder às necessidades $\mathrm{da}$ organização universitária.

Palavras-chave:

Sistema administrativo; gestão acadêmica; Universidade Privada Domingo Savio; Metodologia Agile XP 
INTRODUCCIÓN

Docencia, Investigación y Extensión son consideradas las funciones sustantivas de las universidades, sin embargo, la Gestión de los procesos académicos se convierte en una función estratégica para el logro de todos los objetivos trazados y la interrelación de todas estas funciones. La gestión académica en la Universidad, no solo implica los procesos de enseñanza y aprendizaje que se desarrollan en el aula, sino los procesos administrativos que realiza la institución para concretar su misión y visión. Por tal razón la gestión académica debe articular el diseño de la oferta curricular y la implementación de la oferta académica con el proyecto educativo institucional, apoyar a la gestión de los procesos de enseñanza, al igual que los procesos de aprendizaje de los estudiantes.

Actualmente la gestión académica contribuye a definir el rumbo de una institución educativa, apoya al diseño, la implementación y operación de las decisiones académicas, asimismo contribuye a la evaluación de programas académicos, permitiendo diseñar escenarios para vincular las prácticas educativas con todos los procesos relacionados como oportunidades de acceso, ingreso, permanencia, y promoción de los estudiantes en la institución educativa dentro de los parámetros de la calidad educativa.

Cassasús (2000) y Borjas (2003) coinciden en definir la gestión como al conjunto de acciones enfocadas a la obtención de algún beneficio tomando a las personas que trabajan dentro la institución como el talento profesional activo, a través de procedimientos y herramientas que colaboran al logro de los objetivos, además, es parte del mundo educativo, por tanto este elemento es muy favorable para poder generar una relación entre la gestión y la educación.

El equipo directivo y docente considera que la calidad educativa es la meta, para lo cual planifica, diseña, ejecuta y evalúa la vida educativa con base en los valores, la misión, la visión, los perfiles de formación, los objetivos institucionales tomando en cuenta necesidades detectadas, cambios deseados, expectativas de los usuarios, y con la consecuente implementación de cambios demandados por la sociedad.

La Universidad Privada Domingo Savio (UPDS), es la red universitaria más grande de Bolivia, tiene sus sedes en Santa Cruz, Tarija, Potosí, Cochabamba, La Paz, Trinidad, Sucre y Oruro; cabe destacar que por cumplimiento de normas y seguimiento a reglamentos establecidos, una de sus sedes, logró obtener el reconocimiento a la certificación de sistema de gestión de calidad ISO 9001:2008, este reconocimiento fue el producto de la adecuada gestión de sus procesos, sin embargo la realidad es cambiante y es necesaria una constante revisión para ajustar las acciones a los requerimientos institucionales y del campo de trabajo para los profesionales formados y egresados en esta universidad.

La certificación ISO obtenida impulsa a toda la red universitaria de la UPDS a seguir este ejemplo para lograr los índices de calidad y así obtener este reconocimiento; la realidad es que las otras sedes tienen un sistema de gestión que acusa deficiencias en la gestión del área académica.

Por constituir la gestión académica el eje esencial del servicio educativo, la red universitaria Domingo Savio tiene la necesidad de equipar de herramientas que permitan colaborar, controlar y agilizar la gestión en el área educativa para asegurar el alcance de los objetivos planteados. 
Una de las propuestas más exitosas para optimizar la gestión académica y por ende el servicio educativo es la implementación de un sistema administrativo apoyado en el uso de las tecnologías de la información. El Sistema Administrativo de Información - SAI - (MIS por sus siglas en Inglés Management Information System) mejora los procedimientos académicos, sistematiza la labor de los usuarios, perfecciona la comunicación intra y extra organizacional, incrementa la satisfacción del usuario, disminuye los tiempos de respuesta de los procesos, permite tomar decisiones fundamentadas en información confiable, precisa, oportuna y pertinente. $\mathrm{Al}$ respecto Senn (1997); De Pablos, López, Martín, Tomo y Medina (2004) y Laudon y Laudon (2012) coinciden que un SAI o MIS es un conjunto de recursos, materiales y talento humano interrelacionados de manera dinámica con el propósito de gestionar la información con el propósito de apoyar la toma de decisiones, comprender las situaciones problemáticas y visualizar propuestas con soluciones efectivas.

En la UPDS los procesos de gestión académica está constituidos por cuatro elementos que son: el diseño pedagógico y curricular, prácticas pedagógicas, gestión del aula y seguimiento académico.

1. El diseño pedagógico y curricular se convierten en el sello particular de la organización educativa, contempla las prácticas específicas de enseñanza y aprendizaje, los contenidos y la evaluación (Lavín y del Solar, 2000).

2. Las prácticas pedagógicas están constituidas por los métodos, estrategias, técnicas y recursos para lograr el desarrollo de competencias (Vélez y 2008).

3. La gestión del aula son todos los procesos que se efectúan antes, durante y después del acto pedagógico; incluye planeación, ejecución y evaluación de las actividades que ocurren en el ambiente educativo. (Vélez, Segovia, López, Gonzáles, Valderrama, Fernandes, y Rodríguez, (2008).

4. El seguimiento académico es un proceso administrativo, sistemático $\mathrm{y}$ permanente del desarrollo del acto académico que permiten obtener $\mathrm{y}$ resguardar la información acerca del desempeño del estudiante y del personal docente.

En la red de la UPDS se ponen de manifiesto situaciones que dejan entrever deficiencias en la gestión académica, algunos de estas son: la pérdida de información por deterioro de documentos en el transcurso del tiempo, ocasionando dificultad para recuperar alguna información requerida; el Vicerrectorado y Direcciones de carrera no poseen un procedimiento eficaz para la planificación, ejecución y evaluación de metas e indicadores; carencias en los procedimientos para la valoración del desempeño docente; dificultades para gestionar el cronograma de actividad académica modular y planificación semestral por parte de los jefes de carrera; ausencia de información centralizada acerca del personal la cual es requerida por diferentes instancias en la organización; problemas en la administración y control documental para las defensas de trabajos de grado que realiza la universidad en cumplimiento a los postulantes graduados. 
De acuerdo a lo planteado se puede determinar que la red Universitaria Domingo Savio tiene la necesidad de automatizar los procesos administrativos académicos, ya que presenta dificultades en la gestión académica y se hace necesario agilizar el desenvolvimiento de procedimientos que se desarrollan en la labor del área académica. Por consiguiente se presenta el siguiente enunciado: ¿Cuáles son las características de un sistema administrativo fundamentado en la metodología XP para optimizar la gestión académica en la red universitaria Domingo Savio?; por lo tanto el objetivo que sirve de guía a la investigación es: Desarrollar un sistema administrativo basado en la metodología XP para optimizar la gestión académica de la red Universitaria Domingo Savio.

\section{MÉTODO}

Es una investigación proyectiva de tipo tecnológica ya que se genera una propuesta de un sistema administrativo para atender los resultados del diagnóstico de necesidades de la gestión académica. La población está constituida por 70 profesionales que realiza las operaciones en el área académica en las ocho sedes de la red universitaria Domingo Savio, estos son los jefes de vicerrectorado, los jefes de facultad, asistentes académicos y auxiliares de jefe de carrera. La muestra es aleatoria estratificada de 24 individuos. Las técnicas utilizadas para la recolección de los datos son la entrevista, la encuesta y la observación, en relación los instrumentos quedaron constituidos por un guion de entrevista semiestructurada con 13 preguntas, un cuestionario estructurado con11 ítems, una guía de observación de 6 ítems. El diseño es de campo ya que se recolectan los datos en las diferentes sedes de la UPDS.

La variable de la investigación se resume en el Cuadro 1. 
Cuadro 1. Operacionalización de la variable

\begin{tabular}{|c|c|}
\hline Variable & Definición operacional \\
\hline $\begin{array}{l}\text { Gestión } \\
\text { Académica }\end{array}$ & $\begin{array}{l}\text { La gestión académica, es la esencia } \\
\text { del trabajo de una institución de } \\
\text { Educación Superior, pues señala } \\
\text { cómo se enfocan sus acciones y } \\
\text { procesos para lograr que las tareas } \\
\text { relacionadas con los estudiantes, } \\
\text { docentes y los administrativos } \\
\text { desarrollen las competencias } \\
\text { necesarias para su desempeño } \\
\text { personal, social y profesional, } \\
\text { plasmando la misión de cumplir } \\
\text { con los objetivos previamente } \\
\text { establecidos en el proceso de } \\
\text { planificación estratégica que se } \\
\text { manifiesta en el manual de } \\
\text { procedimientos académicos. }\end{array}$ \\
\hline
\end{tabular}

$\begin{array}{ll}\text { Dimensiones } & \text { Indicadores } \\ \begin{array}{l}\text { Administración de } \\ \text { la gestión }\end{array} & \begin{array}{l}\text { Control de } \\ \text { cumplimiento } \\ \text { manual de } \\ \text { procedimientos }\end{array} \\ & \begin{array}{l}\text { Requerimientos } \\ \text { funcionales }\end{array} \\ & \begin{array}{l}\text { Control de mejora } \\ \text { continua para la } \\ \text { toma de decisiones }\end{array}\end{array}$

Control y

seguimiento de gestión académica

Alcance académico

Estructura
organizativa

Procesos de gestiónmedibles

Prácticas pedagógicas y gestión de aula

\section{RESULTADOS}

La guía de observación permitió detectar que el software de colaboración, que están utilizando UPDS, presenta dificultades técnicas que los usuarios no pueden resolver y en consecuencia no logran obtener la información requerida.

La información de valoración docente no es confiable para realizar el seguimiento a cargo de los jefes de carrera y precisar el reporte del mismo.
Existen fallas con el registro de postulantes a defensas de grado, la generación de actas presenta errores los cuales se deben modificar utilizando herramientas de edición; también se evidenciaron inconsistencias con las fechas de defensas y entrega de actas, errores en nombres $\mathrm{y}$ apellidos de estudiante $\mathrm{o}$ tribunales de evaluación. 
El software, en uso, no está vinculado con el sistema central de la UPDS, mostrando muchas veces datos incorrectos. Es un software que no cumple con los requerimientos por lo tanto los usuarios utilizan herramientas de Office para corregir los problemas por utilizar este software de colaboración.

Los procedimientos que se ejecutan son: (a) Calificaciones: entrega, recepción, introducción, archivo y publicación de centralizadores de notas; (b) Admisión docente; (c) inducción docente; (d) seguimiento y valoración del desempeño docente; (e) planificación semestral y defensa externa.

El cuestionario permitió evidenciar que el $72 \%$ de la muestra reconoce la existencia de un manual de procesos, el 91\% desconocen la existencia de un sistema de información para el seguimiento al desempeño docente. El 58,3\% realizan el informe de valoración del docente mediante la observación en aula, lista de cotejo, autoevaluación; el informe de desempeño se realiza utilizando la herramienta Excel. Los informes no se emiten a tiempo y una de las causas es la carencia de un sistema de información centralizado adaptado a los requerimientos de los usuarios. El 83,3\% mencionan que no cuentan con los procesos referentes a las defensas de los trabajos de grado, es complejo e ineficiente el manejo de la información para realizar informes y reportes requeridos por el personal académico.

\section{El guion de entrevista}

Solo los profesionales de la sede de Potosí utilizan un software de colaboración para realizar la gestión de información, sin embargo declaran dificultades en funcionamiento y errores en los resultados.
El software de colaboración utilizado es independiente del sistema central de la universidad, entonces cuando hay alguna modificación desde la sede central lo tienen que procesar internamente y los datos que son registrados en el sistema central no están vinculados por el software de colaboración, lo que implica que la información que obtienen no es confiable ni oportuna.

Debido que los informes se realiza con herramientas Office, no se tiene un monitoreo de los indicadores y metas establecidas, no existe vigencia de los mismos, se dificulta la obtención de informes entre un rango de fechas, ya que se tienen que hacer integraciones entre archivos de Excel creados por separado o crear pestañas en dicho archivo para imprimir el informe deseado.

Los entrevistados manifiestan que es complicado la manera como realizan las actividades de defensas de grado, tienen plantillas de actas o cronogramas de defensas creadas en Word y Excel de los cuales hacen una copia y modificación de datos cuando algún estudiante es habilitado para su defensa de grado. Y aun es más complicado para las sedes que no poseen el software de colaboración.

\section{Propuesta}

Con base en los resultados del diagnóstico de necesidades se plantea la propuesta de sistema administrativo para optimizar la gestión académica en la UPDS.

El sistema administrativo atiende la gestión de los siguientes procesos académicos: (a) usuario, (b) defensas de grado, (c) seguimiento modular, (d) indicadores, (e) procedimientos. 
Las herramientas tecnológicas utilizadas en la elaboración del sistema administrativo son:

1. Visual Studio.NET es un conjunto de herramientas integrado para la construcción y desarrollo de servicios Web XML, aplicaciones basadas en Microsoft Windows y soluciones Web (Firtman, 2010 y Guérin, 2016).

2. NET Framework es la estrategia base de .NET, administra y ejecuta aplicaciones y servicios Web (Deitel y Deitel, 2007).

3. Modelo Vista Controlador (MVC) El modelo: es un conjunto de clases que describe los datos de las reglas de negocio; la vista: Define como se mostrará la interfaz de usuario; el controlador: es un conjunto de clases que maneja la comunicación del usuario, la aplicación general y la lógica especifica de la aplicación. (Galloway, Wilson, Allen, y Matson, 2014).

4. SQL Server este software define una base de datos, la cual guarda de tal forma que permite un lenguaje de consultas, la finalidad general es almacenar información y permitir a los usuarios la posibilidad de recuperar y actualizar la información (Date, 2011).

5. Boostrap Es el Framework de Twitter que permite crear interfaces web con (Hojas de estilo) CSS y JavaScript que adaptan la interfaz dependiendo del tamaño del dispositivo en el que se visualice (Spurlock, 2013).

6. JQuery es un framework de JavaScript, que funciona en distintos navegadores, el cual ayuda a la creación y depuración de todos los componentes dinámicos en menor tiempo, ofrece una infraestructura con la que se obtiene facilidad de creación de aplicaciones. Es una biblioteca flexible, liviana $\mathrm{y}$ admitido en navegadores modernos (Galloway, Wilson, Allen, y Matson, 2014).

Para el diseño de la propuesta se utilizó la Metodología ágil XP o metodología de programación extrema, la cual se entiende como un enfoque con la participación del cliente en niveles extremos, que contempla cuatro fases: planificación, diseño, codificación y pruebas (Sommerville, 2005 y Pressman, 2010). Ver figura 1. 


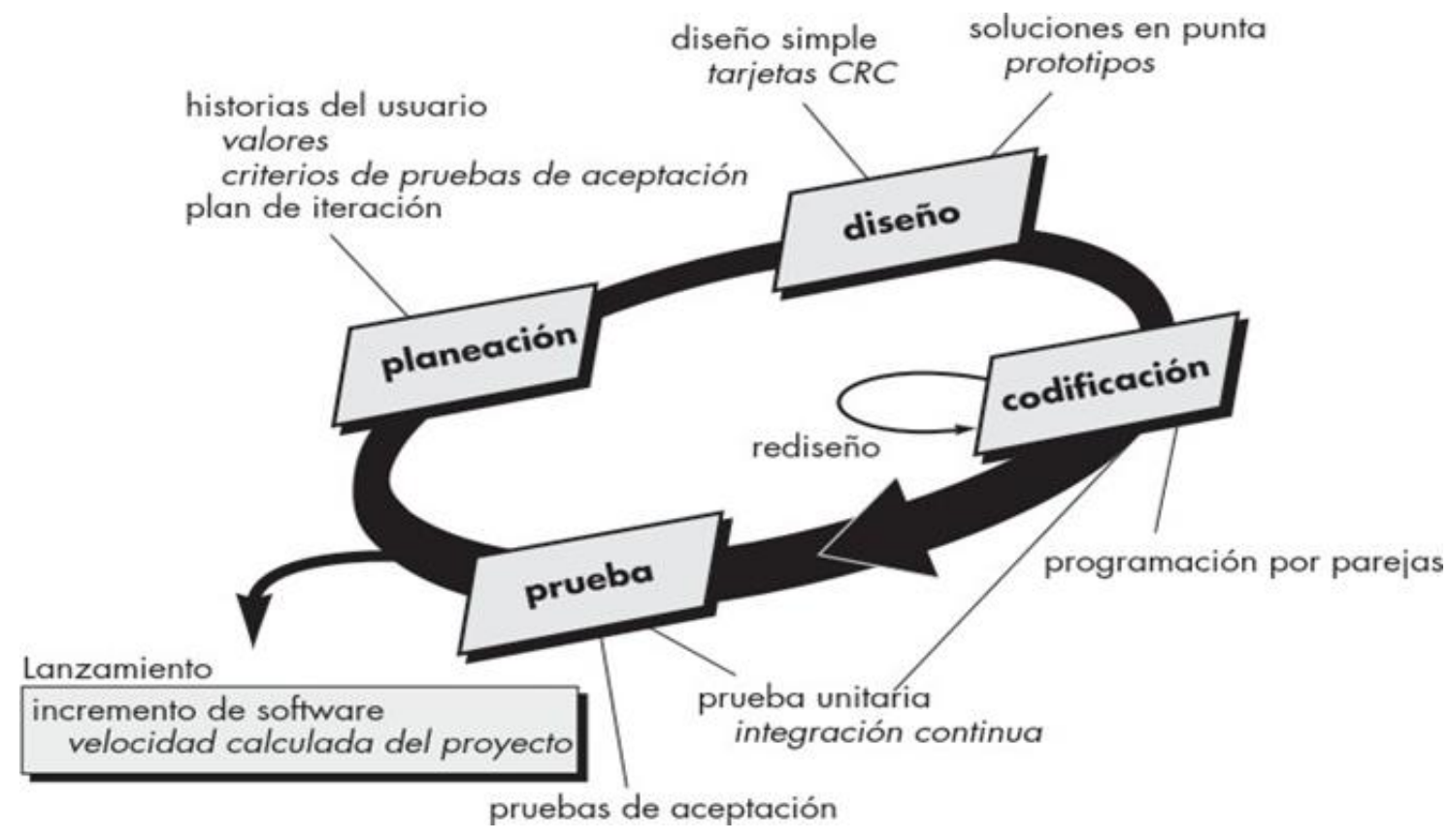

Figura 1. Fases de la programación extrema Metodología Ágil XP (Fuente: Pressman, 2010).

1. Fase de planificación consta de: las historias de usuario, planes de entrega (Release plan), plan de iteraciones (Iteration plan), reuniones diarias de seguimiento (Stand up meeting).

2. Fase de diseño se implementa el diseño más simple y tiene las siguientes actividades: Soluciones spike, Recodificación (refactoring), uso de Metáforas.

3. Fase de codificación le corresponden las siguientes actividades: Disponibilidad del cliente, Uso de estándares, Programación dirigida por las pruebas (Test driven programming), Programación en pares, Integraciones permanentes, Propiedad colectiva del código, Ritmo sostenido.

4. Fase de pruebas en esta fase las actividades involucradas son: Pruebas unitarias, Detección y corrección de errores, Pruebas de aceptación.
Las pruebas de aceptación Según Navarrete (2012) son de vital importancia para el éxito de una iteración y el comienzo de la siguiente, de esta manera el cliente puede conocer el avance del desarrollo del sistema, se denominan las pruebas de caja negra y de caja blanca.

Los roles que se definen en la Metodología Ágil XP son: Programador, cliente, encargado de pruebas (Tester), encargado de seguimiento (Tracker), entrenador (coach), Consultor, Gestor (Big boss).

\section{Desarrollo de la propuesta}

1. Inducción de docentes, de acuerdo a la fase de planificación se construyen los diagramas del proceso de inducción de los docentes que se incorporan a la UPDS y de las actividades involucradas para efectuar la inducción, ver figura 2 y 3. 


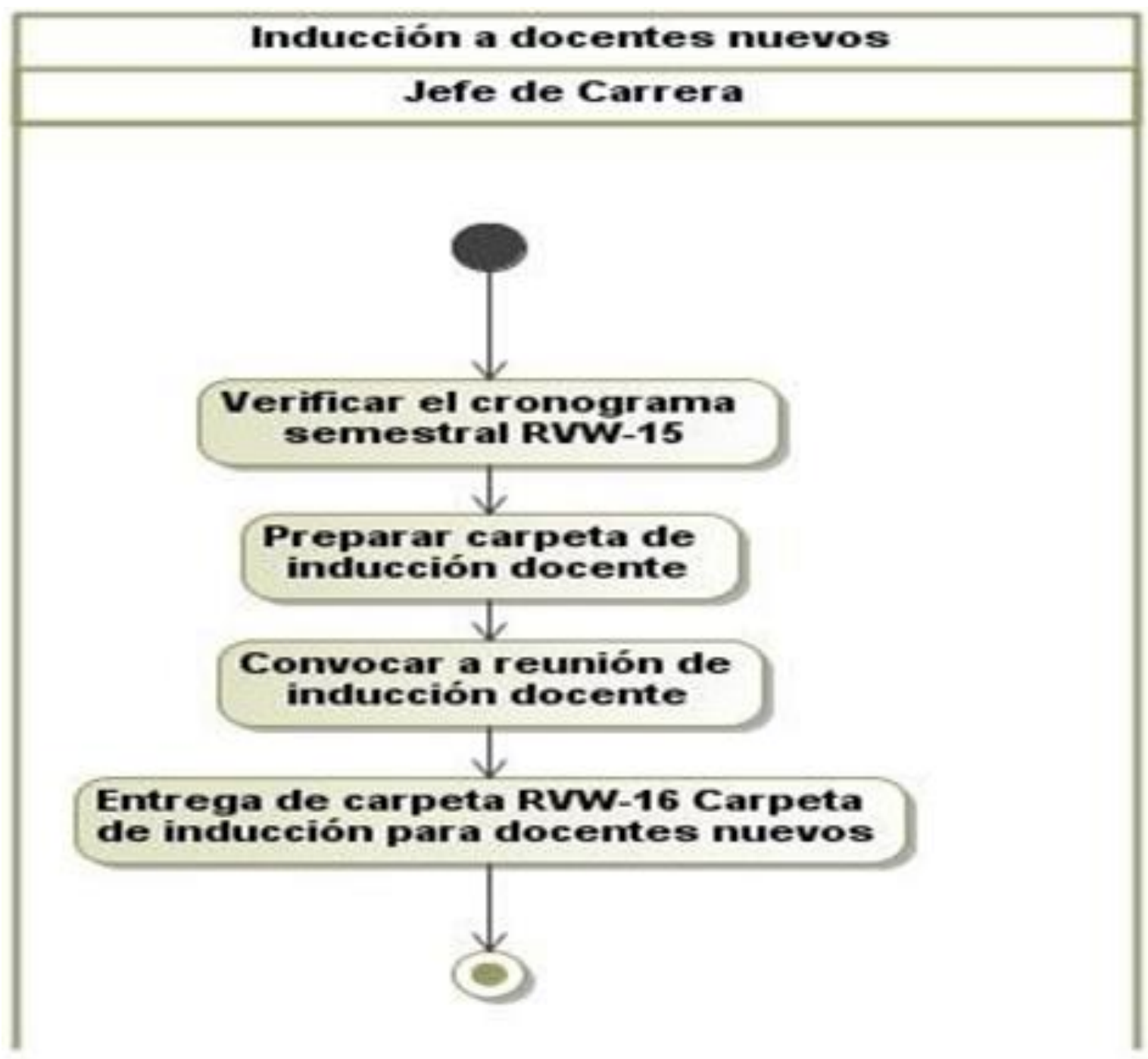

Figura 2. Inducción de nuevos docentes 

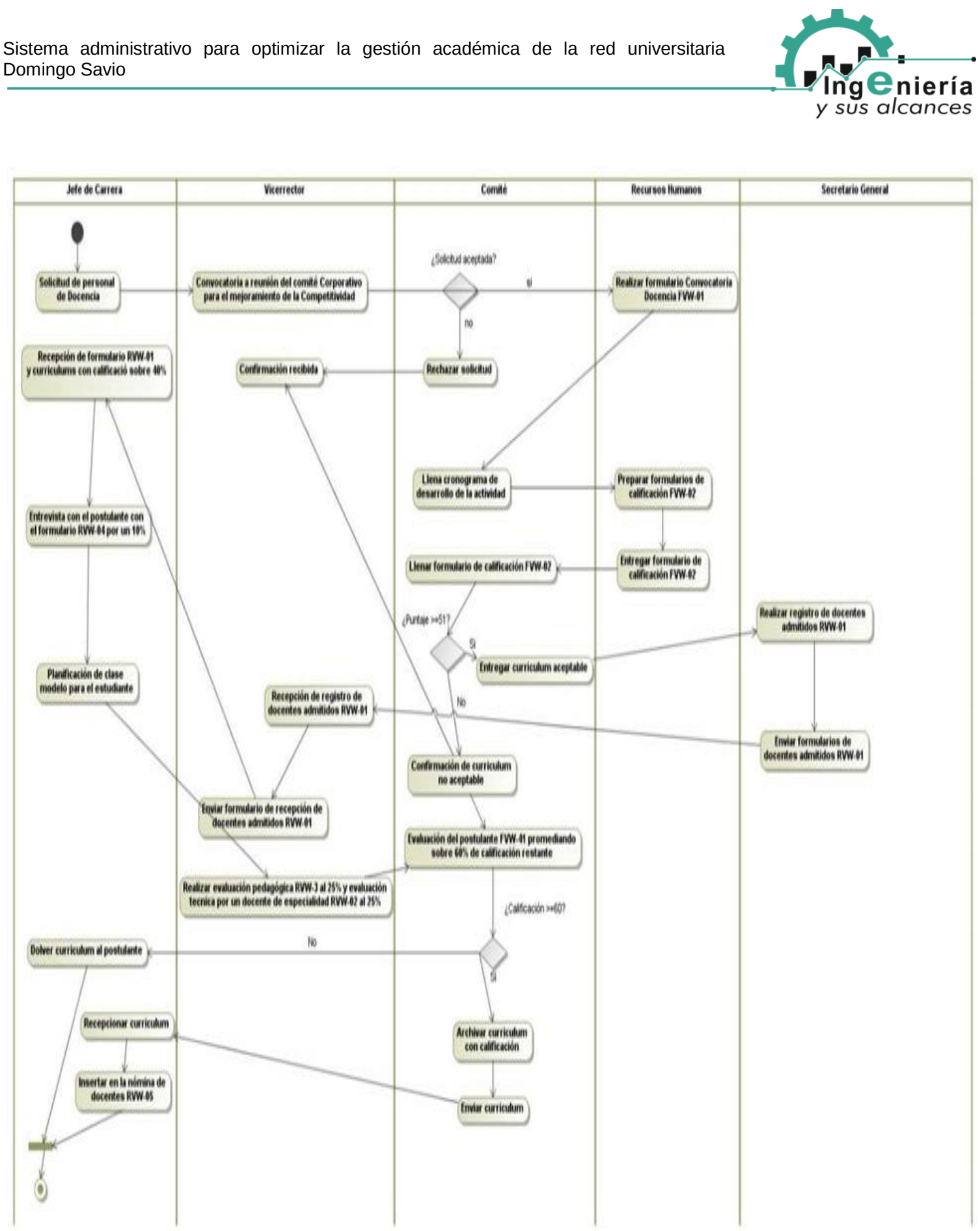

Figura 3. Procedimiento para la de admisión

2. Seguimiento del desempeño docente, para conseguir el sistema de información del desempeño del personal docente se presenta diagramas, ver figura 4. 


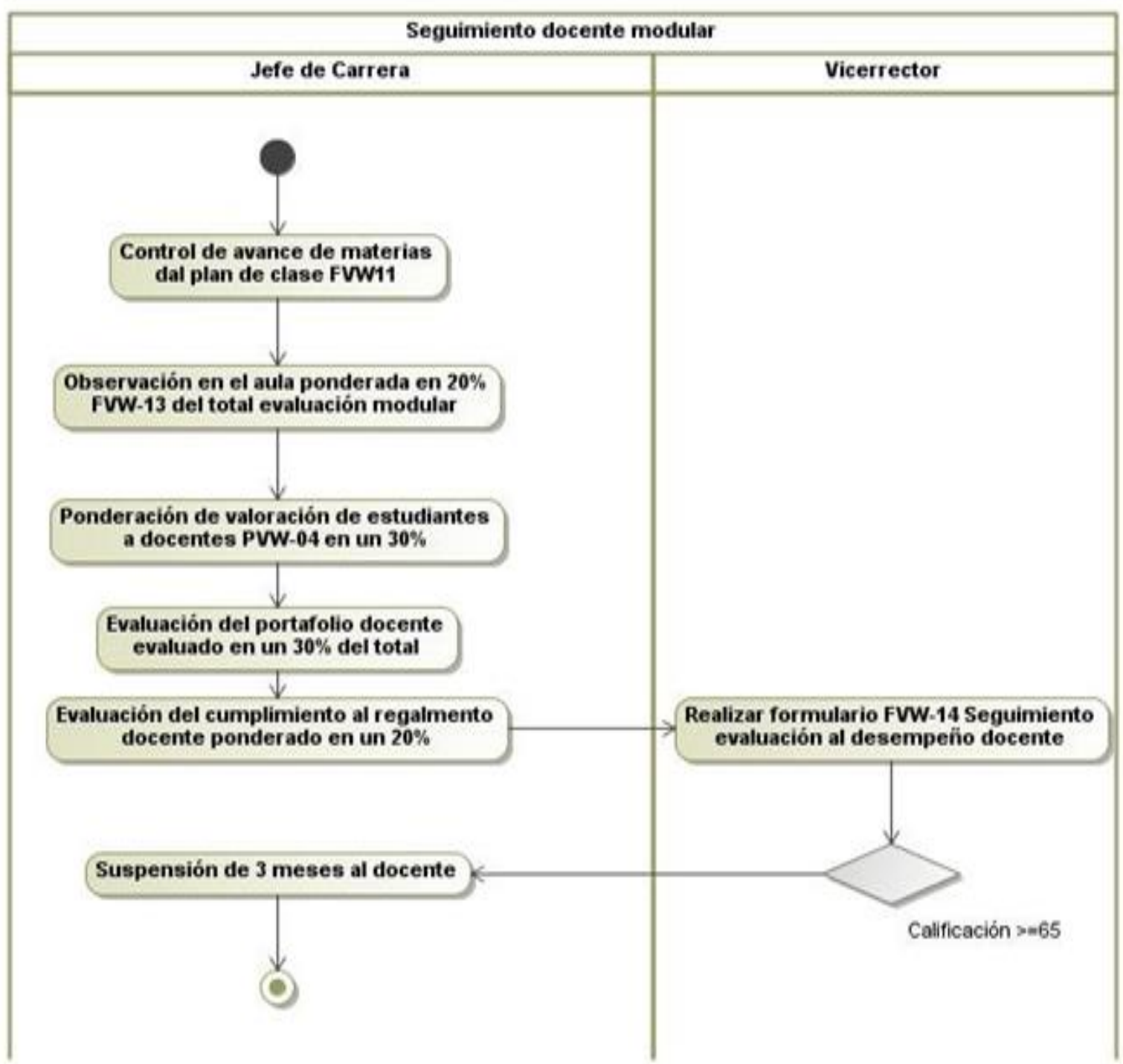

Figura 4. Diagrama de seguimiento del desempeño docente

3. Procedimientos para la defensa de los trabajos de grado, en la figura 5 se resumen las acciones para la organización de las defensas. 


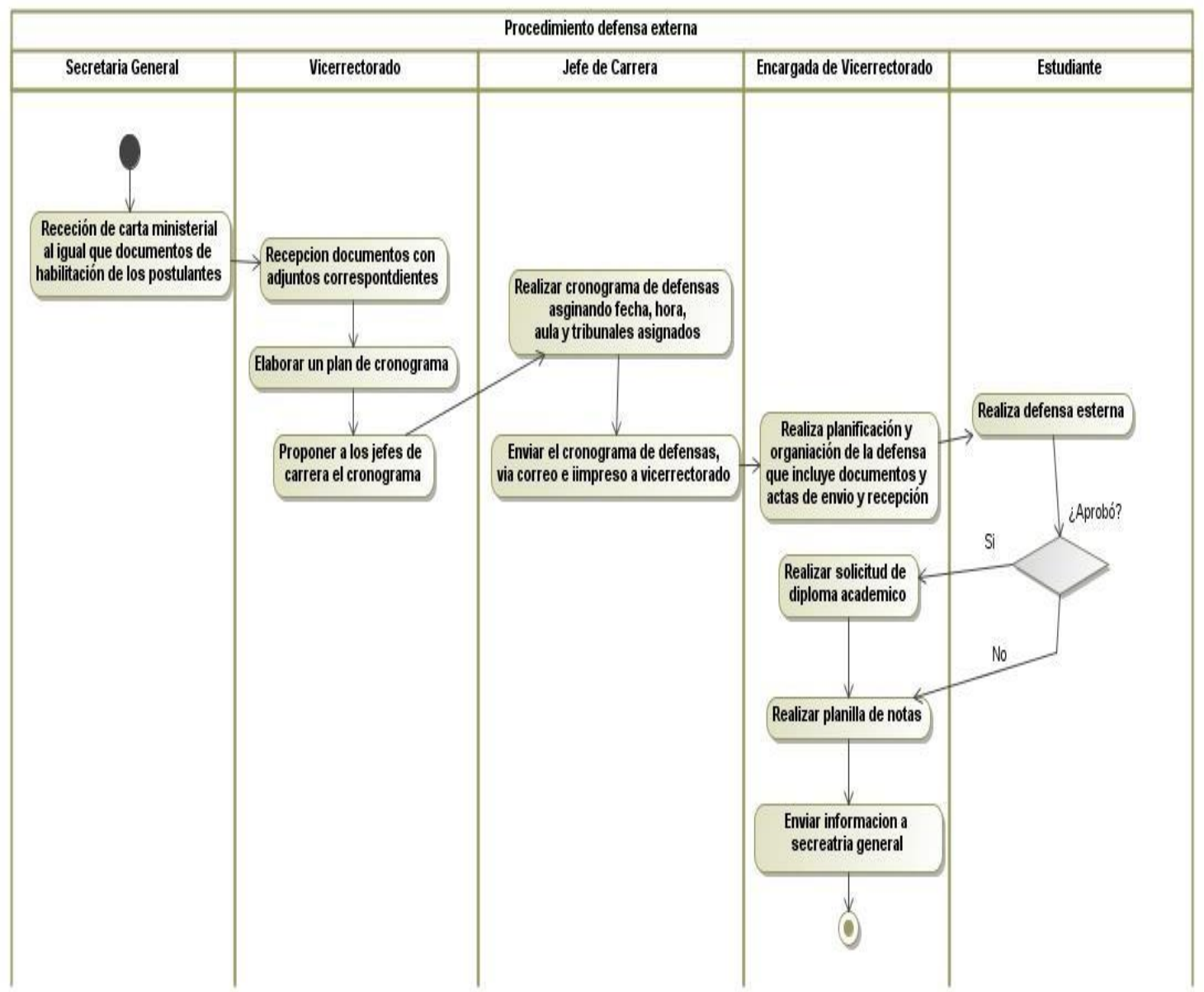

Figura 5. Procedimientos para las defensas de trabajos de grado.

A continuación se muestra una historia de usuario (Ver figura 6) para mostrar la información que contiene, la cual es necesaria para diseñar el sistema administrativo, solo se muestra una historia, para la investigación se construyeron todas las necesarias para el levantamiento de la información y los requerimientos. 


\section{Historia de Usuario}

\begin{tabular}{|l|l|}
\hline Número: 8 & Usuario: Jefe de carrera \\
\hline Nombre Historia: Registro de docentes & Riesgo en desarrollo: Baja \\
\hline Prioridad en Negocio: Media & Iteración asignada: 6 \\
\hline Puntos estimados: 4 & $\begin{array}{l}\text { Programador responsable: Noel Colque } \\
\text { seguidamente seregistre datos de curriculum, por otro lado registrar la calificación que obtuvo } \\
\text { de sus pruebas y exámenes de admisión como docente a la materia que desea postular. Es } \\
\text { importante realizar las exportaciones de informe de curriculum y de la evaluación de admisión } \\
\text { del docente nuevo. }\end{array}$ \\
\hline $\begin{array}{l}\text { Observaciones: filtrar al docente por nombre, apellidos o ci y debe permitir editar los } \\
\text { datoscorrespondientes del seleccionado. }\end{array}$
\end{tabular}

Figura 6. Historia de usuario para el registro de docentes nuevo ingreso.

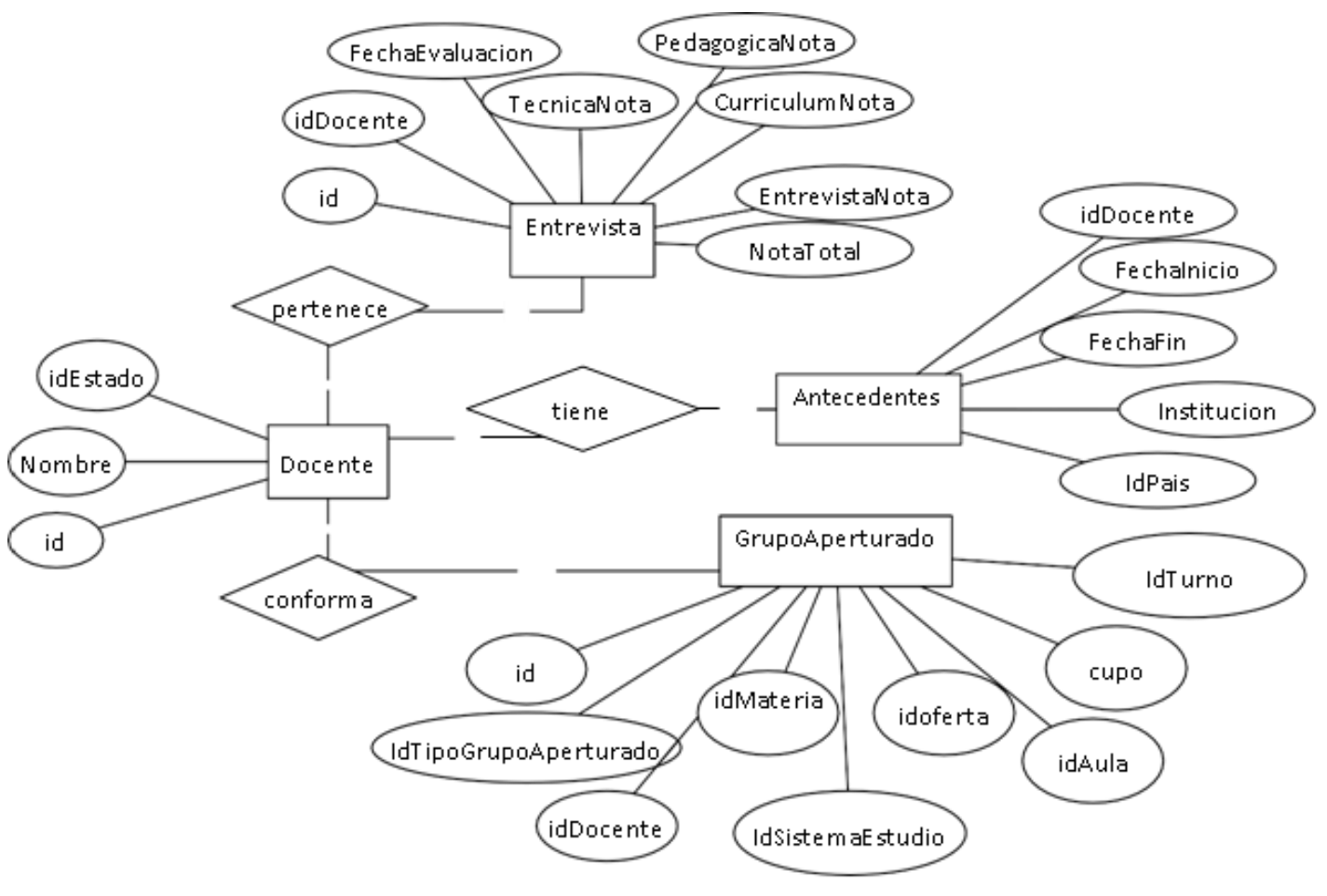

Figura 7. Diagrama de la gestión modular 
A continuación se muestra la imagen de una prueba de caja negra

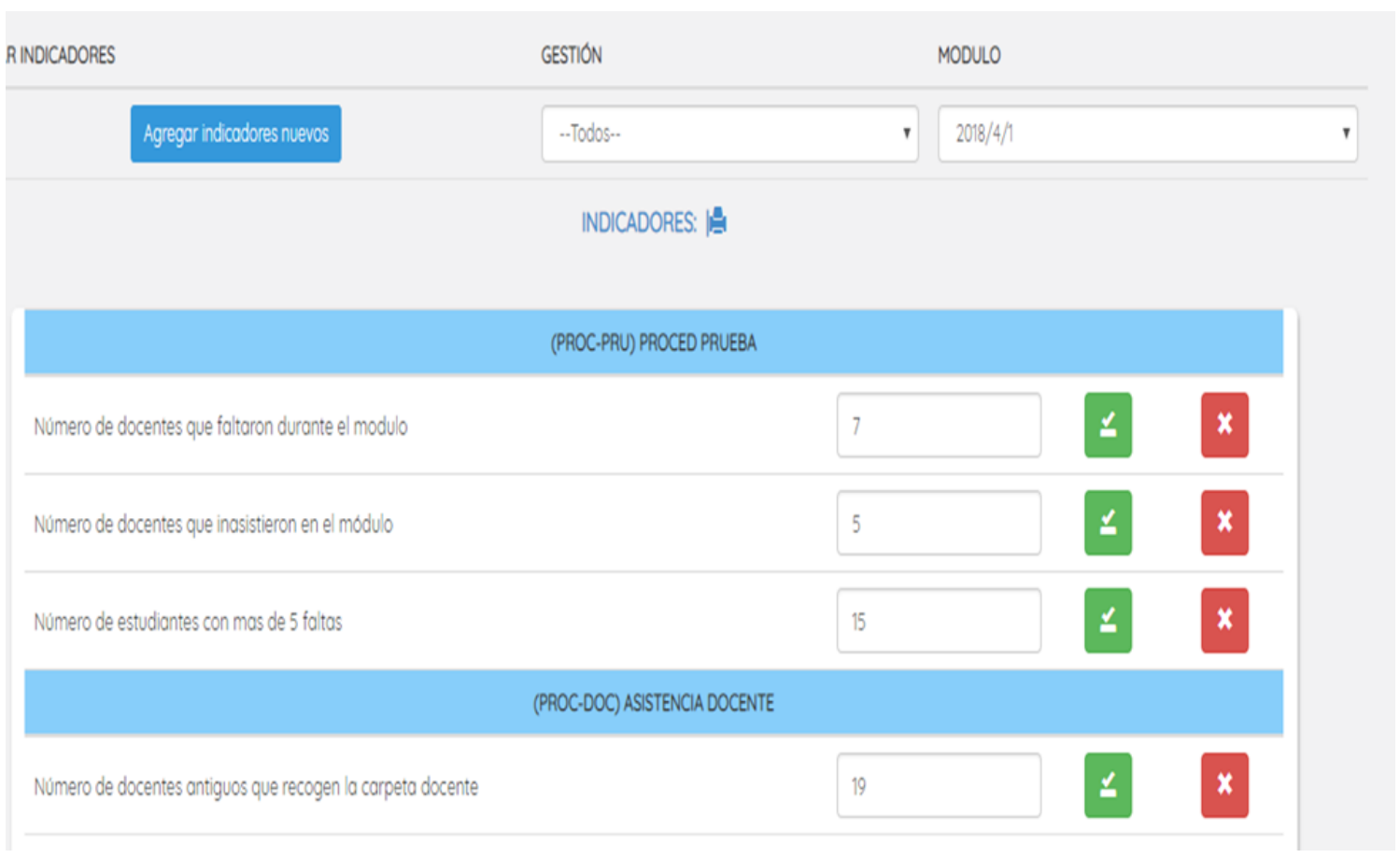

Figura 8. Prueba de caja negra formulario para registrar datos de indicador.

\section{CONCLUSIONES}

La gestión académica resulta ser indispensable para cualquier organización educativa, ya que es un proceso orientado a mejorar los proyectos educativos institucionales, procesos pedagógicos y la dirección correcta de la institución, concretando que la revisión y sistematización de las bases teóricas referidas a las variables de estudio a través de las diversas fuentes de consulta, coadyuvó a comprender y profundizar la problemática planteada y orientar la elaboración de la propuesta.

En el diagnóstico se evidenciaron las carencias de las distintas sedes de la UPDS, no existen procedimientos confiables para el manejo de la información lo cual dificulta el proceso de la gestión académica, evidenciando la necesidad de mejorar el sistema de gestión académica.
Se construyó la propuesta de sistema administrativo aplicando la Metodología Ágil $\mathrm{XP}$, finalizando con las pruebas de aceptación de los usuarios finales.

\section{REFERENCIAS}

Borjas, B. (2003). La Gestión Educativa al servicio de la innovación. Federación Internacional de Fe y Alegría: Caracas

Cassasús, J. (2000). Problemas de la gestión en América Latina: La tensión entre los paradigmas de tipo A y tipo B. Santiago de Chile, Chile: UNESCO

De Pablos, C., López, J. J., Santiago Martín, H., Romo, y Medina, S. (2004). Informática y comunicaciones en la empresa. Madrid, España: ESIC

Deitel, H. M., y Deitel, P. J. (2007). Cómo programar en C\# (2a ed.). México: Pearson Educación

Firtman, M. (2010). Visual Studio.NET Framework 3.5 para profesionales. Buenos Aires: Alfaomega 
Galloway, J., Wilson, B., Allen, S., y Matson, D. (2014). Professional ASP.NET MVC 5

Guérin, B. (2016). ASP.NET en C\# con Visual Studio 2015: Diseño y desarrollo de aplicaciones Web. Barcelona, España: Eni Ediciones

Laudon, K. C., y Laudon, J. P. (2012). Sistemas de información gerencial (12ª. ed.) México: PEARSON

Lavín, S., y del Solar, S. (2000). El proyecto educativo institucional como herramienta de transformación. Santiago de Chile: LOM. Ediciones/PIIE

Navarrete, T. (2012) Análisis comparativo de los métodos de caja negra, Blanca y gris de testing de software, para la Propuesta de un método estándar de testing. Repositorio de la Universidad Pontificia de Ecuador. Disponible: http://repositorio.puce.edu.ec/bitstrea $\mathrm{m} / \mathrm{handle} / 22000 / 6360 / 9.21 .001138 . p d$ f?sequence $=4$ yisAllowed $=y$
Pressman, R. S. (2010). Ingeniería del software: Un enfoque práctico (7a. ed.) México: Mc Graw Hill

Senn, J. A. (1997). Análisis y diseño de sistemas de información (2 $2^{a}$.ed.). México, México: McGraw-Hill

Sommerville, I. (2005). Ingeniería del software (7‥ ed.). Madrid, España: Pearson Educación S.A.

Spurlock, J. (2013). Boostrap Responsive Web Development. United States of America: O'REILLY

Vélez White, C. M., Segovia Ospina, I., López Castro, M., Gonzáles Rivera, A., Valderrama Rengifo, J., Fernandes Cristovao, I., Rodríguez Callazos, M. P. (2008). Guía para el mejoramiento institucional de la evaluación al plan de mejoramiento. Colombia: Ministerio de Educacion Nacional 